\title{
A LOCALLY COMPACT METRIC SPACE IS ALMOST INVARIANT UNDER A CLOSED MAPPING
}

\section{EDWIN DUDA}

1. Introduction. For a given mapping (continuous transformation) of a topological space $X$ onto a topological space $Y$, it has always been of interest to determine what properties of $X$ carry over to $Y$. For example, if $f$ is a closed mapping, then normality [1] and paracompactness [5] are invariants. If $X$ is a metric space and $f$ is a closed mapping, then, as a consequence of results by Vainstein [2], Whyburn [1], and Stone [4], it is known that $Y$ is first countable if and only if each point inverse has a compact frontier. A set $S$ of a space $X$ is said to be a scattered set if every subset of $S$ is a closed subset of $X$. The main result of this paper shows that the image $Y$, of a locally compact metric space $X$ under a closed mapping $f$, minus a scattered set $S$ is also a locally compact metric space. In addition, if $X$ is separable, then $S$ has at most a countable number of points. In case the mapping is also an open mapping, the results are stated in [3], [4], [6] and [7].

2. Notation. For a metric space, $\rho$ is used as the distance function. The frontier of a set $A$ in a metric space $X$ is the closure minus its interior relative to $X$ and is denoted by $\operatorname{Fr} A$. An inverse set of a mapping $f$ of $X$ onto $Y$ is any subset $A$ of $X$ for which $A=f^{-1} f(A)$. Other undefined terms can be extracted from [1], [4] and [5].

\section{Main result.}

THEOREM. Let $f$ be a closed mapping of a locally compact metric space $X$ onto a topological space $Y$. If $S$ is the set of all $y$ in $Y$ such that the frontier of $f^{-1}(y)$ is not compact, then $S$ is a scattered set and $Y-S$ is a locally compact metric space. Moreover, if $X$ is separable, then $S$ is countable.

To facilitate the proof of the main theorem, the following lemma is established.

Lemma. Let $f$ be a closed mapping of $X$ onto $Y$, where $X$ is a locally compact metric space. If $F$ is the union of the point inverses which are not compact, then

(i) F is a closed set;

Presented to the Society, November 16, 1963; received by the editors December $10,1963$. 
(ii) for an arbitrary compact set $K$ in $X$, only a finite number of noncompact point inverses can intersect $K$;

(iii) any inverse set $A$ contained in $F$ is a closed subset of $X$.

Proof. If there are no compact point inverses, then $F=X$ and $F$ is closed. Suppose that $f^{-1}(y)$ is a compact point inverse. Let $U$ be an open set containing $f^{-1}(y)$ and such that $\bar{U}$ is compact. The set $f^{-1} f(X-U)$ is closed and its complement is an open inverse set $V$ containing $f^{-1}(y)$ and contained in $U$. Each compact point inverse is contained in such a set so that $X-F$ is an open set and consequently $F$ is a closed set.

To prove (ii), suppose $K$ is a compact set in $X$ and $f^{-1}\left(y_{n}\right)$ is a sequence of distinct noncompact point inverses, each of which intersects $K$ in a nonempty set. We can assume that $\lim \inf f^{-1}\left(y_{n}\right) \neq \varnothing$ and, hence, if $L=\lim \sup f^{-1}\left(y_{n}\right)$, then $L$ is in some point inverse. Suppose, further, that $L$ is not in any one of the $f^{-1}\left(y_{n}\right)$. If $L$ is a compact set, then there is an open set $W$ containing $L$ with $\bar{W}$ compact. Since no $f^{-1}\left(y_{n}\right)$ is compact, each one has at least one point $x_{n}$ not in $\bar{W}$. The set $\bigcup_{n=1}^{\infty} x_{n}$ is a closed set but $f^{-1} f\left(\bigcup_{n=1}^{\infty} x_{n}\right)=\bigcup_{n=1}^{\infty} f^{-1}\left(y_{n}\right)$ is not a closed set. This is contrary to $f$ being a closed map. Thus, $L$ is not compact. If $L$ is not compact, it contains an infinite sequence of distinct points $y_{n}$, with lim sup $y_{n}=\varnothing$. There is an $f^{-1}\left(y_{n_{1}}\right)$ and a point $x_{1}$ of $f^{-1}\left(y_{n_{1}}\right)$ such that $\rho\left(x_{1}, y_{1}\right)<1$, an $f^{-1}\left(y_{n_{2}}\right)$ and a point $x_{2}$ of $f^{-1}\left(y_{n_{2}}\right)$ such that $\rho\left(x_{2}, y_{2}\right)<1 / 2$, and, in general, an $f^{-1}\left(y_{n_{k}}\right)$ and a point $x_{k}$ of $f^{-1}\left(y_{n_{k}}\right)$ such that $\rho\left(x_{k}, y_{k}\right)<1 / k$. The sequence $x_{k}$ must have $\lim \sup x_{k}=\varnothing$, otherwise $\lim \sup y_{k} \neq \varnothing$. The mapping $f$ is closed and $\bigcup_{k=1}^{\infty} x_{k}$ is a closed set, hence $f^{-1} f\left(\bigcup_{k=1}^{\infty} x_{k}\right)=\bigcup_{k=1}^{\infty} f^{-1}\left(y_{n_{k}}\right)$ is closed. But $\bigcup_{k=1}^{\infty} f^{-1}\left(y_{n_{k}}\right)$ must have a limit point in $K \cap L$ and this gives a contradiction.

To prove (iii), let $A$ be an inverse set contained in $F$. If $A$ is not closed, then there is a sequence $x_{n}$ of distinct points of $A$ converging to a point $x$ not in $A$. Since point inverses are closed sets we can assume that $f^{-1} f\left(x_{i}\right) \cap f^{-1} f\left(x_{j}\right)=\varnothing$ if $i \neq j$. The set $K=U_{x} \bigcup_{n=1}^{\infty} x_{n}$ is compact and an infinite number of distinct noncompact point inverses meet $K$. This contradicts (ii) and therefore $A$ is closed.

Proof of the Theorem. Let $B$ be any subset of $S$. The set $f^{-1}(B)$ is closed in $X$ by (iii) of the Lemma. The mapping $f$ is closed, hence $f f^{-1}(B)=B$ is closed, and $S$ is a scattered set. The set $Y-S$ is the continuous image of $X-f^{-1}(S)$ under $f$ and the mapping $f$ restricted to $X-f^{-1}(S)$ is a closed mapping of $X-f^{-1}(S)$ onto $Y-S$. By a result of Stone [4] (see Theorem 1), we can say that $Y-S$ is metrizable.

To show $Y-S$ is locally compact, let $y \in Y-S$ and suppose that $\operatorname{Fr} f^{-1}(y) \neq \varnothing$. Let $W$ be any open set containing $\operatorname{Fr} f^{-1}(y)$ and such 
that $\bar{W}$ is compact. If $y$ is not interior to $f(W)$, then there exists a sequence of points $y_{n}$ of $(Y-S)-f(W)$ converging to $y$. Since $f$ is a closed mapping, $\lim \sup f^{-1}\left(y_{n}\right) \subset \operatorname{Fr} f^{-1}(y)$. Thus, infinitely many of the $f^{-1}\left(y_{n}\right)$ meet $W$, which in turn implies that infinitely many of the $y_{n}$ are in $f(W)$. This gives a contradiction, hence $y$ is interior to $f(W)$. The interior points of $f(W)$ form an open set whose closure is contained in the compact set $f(\bar{W})$. If $\operatorname{Fr} f^{-1}(y)=\varnothing$, then $\{y\}$ is an open set containing $y$ and $\{\bar{y}\}$ is compact. Thus $Y-S$ is locally compact.

If $X$ is separable, then $X$ is a countable union of compact sets. Only a finite number of point inverses of points in $S$ can meet any one of these compact sets. Thus $f^{-1}(S)$ is a countable union of single point inverses. Therefore $S$ is countable.

4. Examples. To see that $S$ in the theorem can be infinite consider the decomposition of the plane $E_{2}$ into the vertical lines whose equations are $x=n, n$ an integer, and the individual points not on these lines. Let $Y$ be the topological space determined by this upper semicontinuous decomposition of $E_{2}$. The natural mapping of $E_{2}$ onto $Y$ is a closed mapping and $S$ in this case is denumerable.

The following example shows that if local compactness is dropped in the Lemma, then every conclusion must be omitted. Let $X$ be the open unit disk in the plane union the points $(0,1)$ and $(0,-1)$. Consider the decomposition of $X$ into the sets determined by intersecting the graphs of $x=1 / n, n$ a positive integer, and $x=0$, with $X$, and the individual points not on these lines. Again, let $Y$ be the topological space determined by this upper semi-continuous decomposition of $X$. The natural mapping of $X$ onto $Y$ is a closed mapping and no one of the conclusions of the Lemma are valid.

\section{BIBLIOGRAPHY}

1. G. T. Whyburn, Open and closed mappings, Duke Math. J. 17 (1950), 69-74.

2. I. A. Vainstein, On closed mappings in metric spaces, Dokl. Akad. Nauk SSSR (N.S.) 57 (1947), 319-321. (Russian)

3. A. D. Wallace, Some characterizations of interior transformations, Amer. J. Math. 61 (1939), 757-763.

4. A. H. Stone, Metrizability of decomposition spaces, Proc. Amer. Math. Soc. 7 (1956), 690-700

5. E. Michael, Another note on paracompact spaces, Proc. Amer. Math. Soc. 8 (1957), 822-828.

6. G. T. Whyburn, Continuous decompositions, Amer. J. Math. 71 (1949), 218226.

7. V. K. Balanchandran, $A$ mapping theorem for metric spaces, Duke Math. J. 22 (1955), 461-464.

\section{UNIVERSITY OF MiamI}

